\title{
METHODS OF PERTURBATION THEORY IN THE QUALITATIVE ESSENTIAL SPECTRAL ANALYSIS OF DIFFERENTIAL OPERATORS
}

\author{
V.A. EROVENKO \\ Belarusian State University \\ F. Skaryna av. 4, Minsk 220050, Belarus
}

\begin{abstract}
The qualitative properties of perturbed differential equations are investigated. The analogies of the classical Weyl theorem are proved.
\end{abstract}

\section{INTRODUCTION}

In this paper the investigation is conducted of various essential spectra of minimal, maximal and intermediate ordinary differential operators in the Lebesque spaces $L^{p}(a, \infty), 1 \leq p \leq \infty$. These operators are obtained by means of relatively small perturbations of differential operators with constant coefficients of order $n$ by differential operators of the same order. This makes it possible to prove the new analogies of the classic Weyl theorem of invariance of essential spectrum and to obtain the exact formulas for calculating essential spectra of various classes of ordinary differential operators in the Lebesque spaces $L^{p}$. In contemporary mathemetical literature a few assertions are known as a Weyl's theorem. The classic Weyl theorem states that if $A$ and $B$ are self-adjoint and $A-B$ is compact then $\sigma_{e}(A)=\sigma_{e}(B)$, where $\sigma_{e}$ is an essential spectrum of an operator.

\section{FORMULATION OF THE PROBLEM}

Let $T$ be a closed linear operator densely defined on a complex Banach space. Essential spectra of an operator $T$ could be defined as the complement in a complex plane $\mathbf{C}$ of a set defined by various Fredholm properties of family of 
operators $T-\lambda I$ :

$$
\begin{gathered}
\sigma_{e k}(T):=\mathbf{C} \backslash \Delta_{k}(T), \quad k=1,2,3,4,5, \\
\sigma_{e 2}^{+}(T):=\mathbf{C} \backslash \Phi^{+}(T) \quad \text { and } \quad \sigma_{e 2}^{-}(T):=\mathbf{C} \backslash \Phi^{-}(T),
\end{gathered}
$$

where $\Delta_{1}(T):=\{\lambda \in \mathbf{C}: \overline{R(T-\lambda I)}=R(T-\lambda I)\}, \quad \Phi^{+}(T):=\{\lambda \in$ $\left.\Delta_{1}(T): \operatorname{nul}(T-\lambda I)<\infty\right\}, \quad \Phi^{-}(T):=\left\{\lambda \in \Delta_{1}(T): \operatorname{def}(T-\lambda I)<\right.$ $\infty\}, \quad \Delta_{2}(T):=\Phi^{+}(T) \cup \Phi^{-}(T)=s-\Phi(T), \quad \Delta_{3}(T):=\Phi^{+}(T) \cap \Phi^{-}(T)=$ $\Phi(T), \quad \Delta_{4}(T):=\left\{\lambda \in \Delta_{3}(T): \operatorname{ind}(T-\lambda I)=0\right\}, \quad \Delta_{5}(T):=\left\{\lambda \in \Delta_{4}(T):\right.$ a deleted neighbourhood of $\lambda$ lies in the resolvent set $\rho(T)\}$.

Each of the sets $\sigma_{e k}(T), k=\overline{1,5}, \sigma_{e 2}^{+}(T)$ and $\sigma_{e 2}^{-}(T)$ has been reffered to as the essential spectrum of $T$ accoding to (1) Goldberg, (2) Kato, $\left(2^{+}\right)$ Wolf, (2- ) Gustafson-Weidmann, (3) Fredholm, (4) Weyl or Schechter, (5) Browder. It is clear, that $\sigma_{e k}(T) \subseteq \sigma_{e l}(T)$ for $k \leq l$ and $\sigma_{e 2}(T) \subseteq \sigma_{e 2}^{ \pm}(T) \subseteq$ $\sigma_{e 3}(T)$, were the inclusion might be proper. The essential spectra $\sigma_{e k}(T), k=$ $1,2,2^{ \pm}, 3,4,5$, can be described by other equivalent means [1-3].

Let consider a formal differential expression

$$
\tau:=\sum_{k=0}^{n} a_{k}(t) D^{k}, \quad a \leq t<\infty, \quad-\infty<a<\infty
$$

where $a_{k}(t)$ are complex valued functions such that $a_{k}(t) \in C^{k}[a, \infty), a_{n}(t) \neq$ $0,1 / a_{n}, a_{k} \in L^{\infty}(a, \infty), 0 \leq k \leq n$, and $D:=d / d t$. Denote by $T(\tau, p,[a, \infty))$ a maximal operator corresponding to $(\tau, p,[a, \infty))$ which is defined on $L^{p}(a, \infty)$ as follows:

$$
D[T(\tau, p,[a, \infty))]:=\left\{f: f^{(n-1)} \in A C_{l o c}[a, \infty) ; f, \tau f \in L^{p}(a, \infty)\right\}
$$

where $A C_{l o c}[a, \infty)$ is the set of complex valued functions $f$, absolutely continuos on each compact subinterval from $[a, \infty)$ and

$$
T(\tau, p,[a, \infty)) f:=\tau f \quad \text { for } \quad f \in D[T(\tau, p,[a, \infty))] .
$$

We denote by $T_{0}(\tau, p,[a, \infty))$ a minimal operator defined on $L^{p}(a, \infty)$ for $1 \leq p<\infty$ as a closure of restriction of a maximal operator $T(\tau, p,[a, \infty))$ on a set of functions from $D[T(\tau, p,[a, \infty))]$, having compact support in $(a, \infty)$, and for $1<p \leq \infty$ defined by a Banach conjugate $T^{\prime}\left(\tau^{*}, p^{\prime},[a, \infty)\right)$, where $\tau^{*}$ is the formally conjugated differential operation $\tau^{*} g:=\sum_{k=0}^{n}(-1)^{k} D^{k}\left(a_{k} g\right)$, and $1 / p+1 / p^{\prime}=1$ if $1<p<\infty ; p^{\prime}=\infty$ if $p=1 ; p^{\prime}=1$ if $p=\infty$. Various properties of essential spectra of minimal and maximal ordinary differential operators are investigated in [4-7]. 


\section{MAIN RESULTS}

Theorem 1. Let $S(\tau, p,[a, \infty)),-\infty<a<\infty$, be a closed linear differential operator in $L^{p}(a, \infty), 1 \leq p \leq \infty$, which is an extention of the minimal operator $T_{0}(\tau, p,[a, \infty))$ and a restriction of the maximal operator $T(\tau, p,[a, \infty))$ generated by differential operation $\tau(1)$ with smooth coefficients $a_{k}(t), 1 / a_{n} \in$ $L^{\infty}(a, \infty), 0 \leq k \leq n$,

$$
T_{0}(\tau, p,[a, \infty)) \subseteq S(\tau, p,[a, \infty)) \subseteq T(\tau, p,[a, \infty)) .
$$

Then for any $b \in(a, \infty)$ and five versions of essential spectra of differential operators $S(\tau, p,[a, \infty))$ and $S(\tau, p,[b, \infty))$ the equalities hold:

$$
\sigma_{e k}[S(\tau, p,[a, \infty))]=\sigma_{e k}[S(\tau, p,[b, \infty))], \quad k=1,2,2^{ \pm}, 3 .
$$

For the Weyl essential spectrum $\sigma_{e 4}$ of minimal and maximal differential operators the following equalities hold:

$$
\begin{aligned}
& \sigma_{e 4}\left[T_{0}(\tau, p,[a, \infty))\right]=\sigma_{e 4}\left[T_{0}(\tau, p,[b, \infty))\right], \\
& \sigma_{e 4}[T(\tau, p,[a, \infty))]=\sigma_{e 4}[T(\tau, p,[b, \infty))] .
\end{aligned}
$$

We denote by $B(\nu, p,[a, \infty))$ (respectively $\left.B_{0}(\nu, p,[a, \infty))\right)$ for $-\infty<a<\infty$ a maximal (minimal) differential operator generated in $L^{p}(a, \infty), 1 \leq p<\infty$, by the formal differential operation

$$
\nu:=\sum_{k=0}^{n-1} b_{k}(t) D^{k}, \quad a \leq t<\infty
$$

where complex valued functions $b_{k} \in C^{k}[a, \infty), 0 \leq k \leq n-1$, and by $T(\tau+$ $\nu, p,[a, \infty)$ ) (respectively $T_{0}(\tau+\nu, p,[a, \infty))$ ) a maximal (minimal) operator generated in $L^{p}(a, \infty),-\infty<a<\infty, 1 \leq p<\infty$, by the formal differential operation $\tau+\nu$, where $\tau$ and $\nu$ are defined by the formulae (1) and (6). The operators $B(\nu, p,[a, \infty)), B_{0}(\nu, p,[a, \infty))$ and $T(\tau+\nu, p,[a, \infty)), T_{0}(\tau+$ $\nu, p,[a, \infty))$ are defined likewise the maximal and minimal differential operator denerated by the operation $\tau$.

TheOREM 2. The maximal differential operator $B(\nu, p,[a, \infty)$ ) (the minimal differential operator $B_{0}(\nu, p,[a, \infty))$ ) generated by $\nu(2)$ in $L^{p}(a, \infty)$ is $T(\tau, p,[a, \infty))$-bounded (respectively $T_{0}(\tau, p,[a, \infty))$-bounded) for the differential operation $\tau(1),-\infty<a<\infty$ and $1 \leq p<\infty$ if

$$
\sup _{m \leq s<\infty} \int_{s}^{s+1}\left|b_{k}(t)\right|^{p} d t \rightarrow 0 \quad \text { as } \quad m \rightarrow \infty, \quad 0 \leq k \leq n-1 .
$$


In case of fairly large $a \in(0, \infty)$ for maximal and minimal differential operators considered the equalities hold

$$
\begin{gathered}
T(\tau+\nu, p,[a, \infty))=T(\tau, p,[a, \infty))+B(\nu, p,[a, \infty)), \\
T_{0}(\tau+\nu, p,[a, \infty))=T_{0}(\tau, p,[a, \infty))+B_{0}(\nu, p,[a, \infty)),
\end{gathered}
$$

and a relative bound of differential operators $B(\nu, p,[a, \infty))$ and $B_{0}(\nu, p,[a, \infty))$ is strictly less than unity.

Using theorem 2 and the theorem of essential spectra of maximal and minimal ordinary differential operators with constant coefficients [5] one can find exact formulas for essential spectra of differential operators with almost constant coefficients, i.e. differential operators with variable coefficients tending to constant ones at infinity.

Let us consider a formal differential operation

$$
\mu:=\tau+\nu=\sum_{k=0}^{n} a_{k} D^{k}+\sum_{k=0}^{n-1} b_{k}(t) D^{k}, \quad a \leq t<\infty,
$$

where $a_{k}$ are complex numbers, $\tau$ is a differential operation of type (1) with constant coefficients and $b_{k}(t)$ are complex valued functions such that $b_{k} \in$ $C^{k}(a, \infty), k=\overline{0, n}$.

Theorem 3. Let the coefficients $b_{k}(t), 0 \leq k \leq n-1$ in the differential operation $\mu$ (4) satisfy the integral conditions (3).

Then for the essential spectra of minimal $T_{0}(\mu, p,[a, \infty))$, maximal $T(\mu, p,[a, \infty))$ operators generated by $\mu(4)$ in $L^{p}(a, \infty),-\infty<a<\infty, 1 \leq$ $p<\infty$, and for the closed differential operator $S(\mu, p,[a, \infty))$, which is an extension of minimal and a restriction of maximal operators, as well as for similar operators defined by the differential operations $\tau$ (1) with constant coefficients and $\nu(2)$, the following equalities hold

$$
\begin{aligned}
\sigma_{e k}[S(\mu, p,[a, \infty))] & =\sigma_{e k}[S(\tau, p,[a, \infty))+S(\nu, p,[a, \infty))] \\
& =\sigma_{e k}[S(\tau, p,[a, \infty))], \quad k=1,2,2^{ \pm}, 3 \\
\sigma_{e k}\left[T_{0}(\mu, p,[a, \infty))\right] & =\sigma_{e k}\left[T_{0}(\tau, p,[a, \infty))+T_{0}(\nu, p,[a, \infty))\right] \\
& =\sigma_{e k}\left[T_{0}(\tau, p,[a, \infty))\right], \quad k=4,5, \\
\sigma_{e k}[T(\mu, p,[a, \infty))] & =\sigma_{e k}[T(\tau, p,[a, \infty))+T(\nu, p,[a, \infty))] \\
& =\sigma_{e k}[T(\tau, p,[a, \infty))], \quad k=4,5 .
\end{aligned}
$$

These equalities are the generalizations of the classic Weyl theorem. 
In theorem 2 of [5] the exact formulae for finding all essential spectra of maximal and minimal differential operators with constant coefficients were abtained.

THEOREM 4. Essential spectra of differential operators generated by the operation $\mu$ (4) and defined in theorem 3 can be calculated by

$$
\begin{gathered}
\sigma_{e k}[S(\mu, p,[a, \infty))]=\quad \begin{array}{l}
\sigma_{e 2}^{ \pm}[S(\mu, p,[a, \infty))]=\{P(\lambda): \operatorname{Re} \lambda=0\}, \\
k=\overline{1,3},
\end{array} \\
\sigma_{e k}\left[T_{0}(\mu, p,[a, \infty))\right]=\begin{array}{l}
\sigma\left[T_{0}(\mu, p,[a, \infty))\right]=\{P(\lambda): \operatorname{Re} \lambda \geq 0\}, \\
k=4,5,
\end{array} \\
\sigma_{e k}[T(\mu, p,[a, \infty))]=\sigma[T(\mu, p,[a, \infty))]=\{P(\lambda): \operatorname{Re} \lambda \leq 0\}, k=4,5,
\end{gathered}
$$

where $P$ is a polynomial corresponding the operation with constant coefficients $\tau(1)$

$$
P(t):=\sum_{k=0}^{n} a_{k} t^{k}
$$

Let us consider a differential operation $\mu$ of more general type, namely, as a perturbation of $\tau$ by a differential operation of the same order $n$, i.e.

$$
\tilde{\mu}:=\sum_{k=0}^{n}\left(a_{k}+b_{k}(t)\right) D^{k}, \quad a \leq t<\infty,
$$

where $a_{k}$ are complex numbers and complex valued functions of real argument $b_{k} \in C^{k}[a, \infty)$, for $k=\overline{0, n}$.

THEOREM 5. Let the conditions $b_{n}, 1 /\left(a_{n}+b_{n}\right) \in L^{\infty}(a, \infty)$ hold for coefficients of the differential operation $\tilde{\mu}(8)$ and coefficients $b_{k}(t), 0 \leq k \leq n$, satisfy conditions of tending to 0 at infinity (3).

Then the following generalizations of the classic Weyl theorem hold

$$
\begin{gathered}
\sigma_{e k}[S(\tilde{\mu}, p,[a, \infty))]=\sigma_{e k}[S(\tau, p,[a, \infty))], \quad k=1,2,2^{ \pm}, 3, \\
\sigma_{e k}\left[T_{0}(\tilde{\mu}, p,[a, \infty))\right]=\sigma_{e k}\left[T_{0}(\tau, p,[a, \infty))\right], \quad k=4,5, \\
\sigma_{e k}[T(\tilde{\mu}, p,[a, \infty))]=\sigma_{e k}[T(\tau, p,[a, \infty))], \quad k=4,5 .
\end{gathered}
$$

for the minimal $T_{0}(\tilde{\mu}, p,[a, \infty))$, maximal $T(\tilde{\mu}, p,[a, \infty))$ and intermediate $S(\tilde{\mu}, p,[a, \infty))$ differential operators generated in $L^{p}(a, \infty),-\infty<a<\infty, 1 \leq$ $p<\infty$, by the operation $\tilde{\mu}$ (8) Besides, for differential operators defined by the operations $\tilde{\mu}(8)$ the formulas for essential spectra $(5)-(7)$ hold. 
Since theorem 2 holds for the differential operation $\tau$ (1) with variable coefficients, considering the conditions on the coefficients $b_{k}(t), 0 \leq k \leq n$, (3) it sufficent to investigate the particular case of the differential operation $\tilde{\mu}(8)$ with the coefficients $b_{k}(t)=0,0 \leq k \leq n-1$

$$
\hat{\mu}:=\tau+b_{n}(t) D^{n}=\left(a_{n}+b_{n}(t)\right) D^{n}+\sum_{k=0}^{n-1} a_{k} D^{k} .
$$

Hence firstly we prove the assertion of the theorem for differential operators with $\hat{\mu}(9)$ and then consider the general differential operation $\tilde{\mu}(8)$ as a relatively small perturbation of the differential operation $\hat{\mu}(9)$. In order to do so it sufficet to present the differential operation $\hat{\mu}(9)$ as

$$
\hat{\mu}-\lambda=\left(1+\frac{b_{n}(t)}{a_{n}}\right)(\tau-\lambda)-\sum_{k=0}^{n-1} \frac{b_{n}(t)}{a_{n}} a_{k} D^{k}+\frac{b_{n}(t)}{a_{n}} \lambda .
$$

THEOREM 6. For the essential spectra of minimal $T_{0}(\tilde{\mu}, \infty,[a, \infty))$, maximal $T(\tilde{\mu}, \infty,[a, \infty))$ and intermediate $S(\tilde{\mu}, \infty,[a, \infty))$ differential operators generated by the formal differential operation $\tilde{\mu}$ (8) in space $L^{\infty}(a, \infty),-\infty<$ $a<\infty$, with coefficients satisfying the conditions of theorem 5 and with their derivatives satisfying

$$
\sup _{m \leq s<\infty} \int_{s}^{s+1}\left|b_{k}^{(i)}(t)\right|^{p} d t \rightarrow 0 \quad \text { as } \quad m \rightarrow \infty, \quad 0 \leq i \leq k, 0 \leq k \leq n
$$

the formulas $(5)-(7)$ hold for differential operators in case $p=\infty$.

Proof. It is sufficent to apply theorem 5 to differential operators generated in space $L^{1}(a, \infty)$ by the formal cojugated differential operation

$$
(\tilde{\mu})^{*} f:=\sum_{k=0}^{n}(-1)^{k} D^{k}\left(\left(a_{k}+b_{k}(t)\right) f\right)
$$

and then using the formulas of duality (see, for instance, [4]) proceed to differential operators defined by the operation $\tilde{\mu}(8)$ in space $L^{\infty}(a, \infty)$.

Theorems 3-6 generalize the results of [1] for Fredholm and Goldberg essential spectra of maximal operators in space $L^{p}(0, \infty)$ as well as the results of [8] for various essntial spectra of ordinary differential operators in Hilbert space. We would like to note books $[9,10]$ on localization of essential spectrum of ordinary self-adjoint differential operators with variable coefficients. 


\section{REFERENCES}

[1] Goldberg S., Unbounded linear operators. Theory and application. New York, (1966).

[2] Schechter M., Spectra of partial differential operators. North-Holland, Amsterdam, (1971).

[3] Edmunds D.E., Evans W.D. Spectral theory and differential operators. Oxford, (1987).

[4] Erovenko V.A. //Differential equations, 32, No 8 (1996), P. 1024 - 1034.

[5] Erovenko V.A. //Differential equations, 32, No 9 (1996), P. $1162-1170$.

[6] Erovenko V.A. //Differential equations, 33, No 7 (1977), P. $867-875$.

[7] Erovenko V.A. $L^{p}$-essential spectral theory of ordinary differential operators with almost constsnt coefficients. Matematički Vestnik 50 (1998) (will appear).

[8] Evans W.D., Lewis R.T., Zettl A. Non-self-adjoint operators and their essential spectra. Lect. Notes Math., N 1032 (1982), P. 123 - 160.

[9] Glazman I.M. Direct methods of qualitative spectral analisis of singular differential operators. Jerusalem, (1965).

[10] Müller-Pfeiffer E. Spectral theory of ordinary differential operators. John Wiley \& Son, New York, (1981). 